\title{
Hyperammonemic encephalopathy due to carbonic anhydrase VA deficiency
}

INSERM

\section{Source}

INSERM. (1999). Orphanet: an online rare disease and orphan drug data base. Hyperammonemic encephalopathy due to carbonic anhydrase VA deficiency. ORPHA:401948

A rare, hereditary inborn error of metabolism characterized by an acute onset of encephalopathy in infancy or early childhood. Apart from these episodic acute events, the disorder shows a relatively benign course. Multiple metabolic abnormalities are present, including metabolic acidosis, respiratory alkalosis, hypoglycemia, increased serum lactate and alanine. 